\title{
Metastatic malignant melanoma of duodenum: the tip of the iceberg
}

\author{
S. Leite, A. Rodrigues, J. Pinto, M. Afonso, R. Veloso, J. Carvalho and J. Fraga \\ Gastroenterology and Hepatology Department. Centro Hospitalar Vila Nova Gaia/Espinho. Vila Nova de Gaia, Portugal
}

\section{CASE REPORT}

A 47-year-old male patient was referred to our gastroenterology department, to investigate multiple and large conglomerate mediastinal adenopathies.

He reported a 6-month history of anorexia, weight loss, hoarseness, decrease in visual acuity, and several pigmented skin nodules scattered over the whole body with an average size of $2 \mathrm{~cm}$. More recently, he presented with diffuse abdominal pain, nausea and vomiting.

Upper endoscopy showed at the duodenal bulb and second part of duodenum, several sessile erythematous polyps, between 5 and $15 \mathrm{~mm}$ with central ulceration (Fig. 1). The biopsy specimen revealed metastatic malignant melanoma. Immunohistochemistry stains showed neoplastic cells positive for S-100 protein (Fig. 2).

Cutaneous investigation confirmed a very aggressive malignant melanoma (Fig. 3), with disseminated metastasis, including intra-orbital, thyroid, bronchial, lymphatic, heart, pancreatic, peritoneal and bone metastasis.

Clinical management relied on symptomatic therapy only.

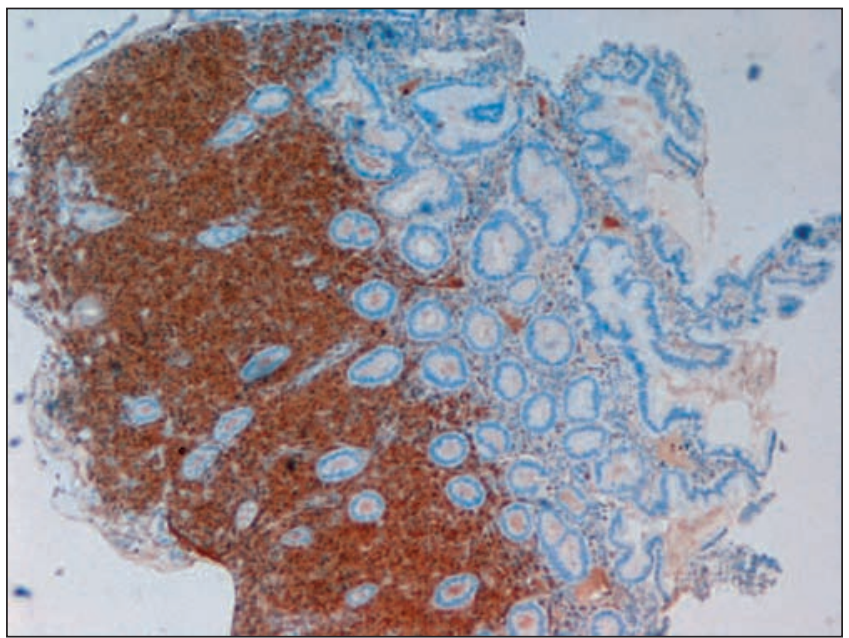

Fig. 2. Histology with immunostaining of duodenal tissue showing positivity for S-100 antibody in the neoplastic cells.

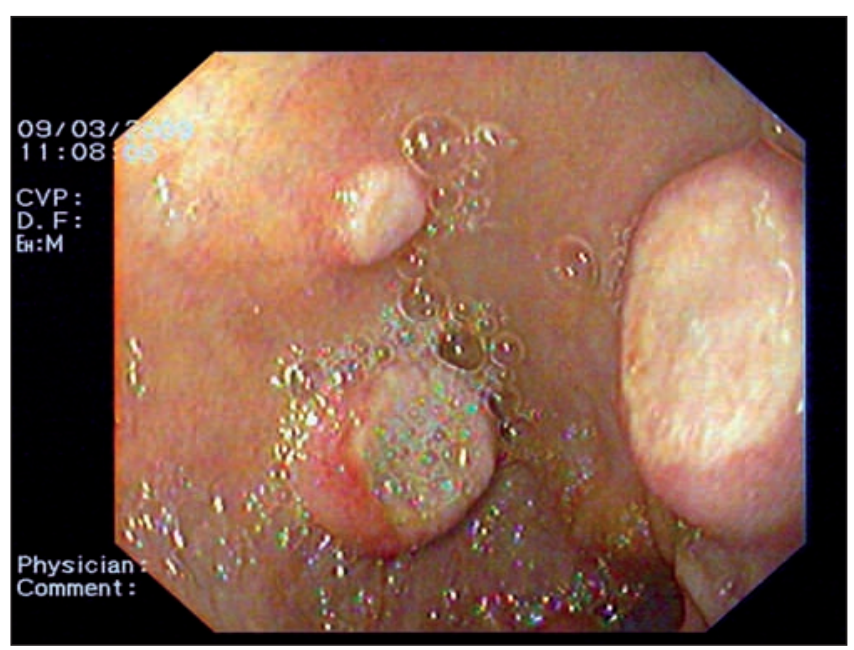

Fig. 1. Endoscopic image of the duodenal bulb and second part of duodenum: several sessile polyps with central depression.

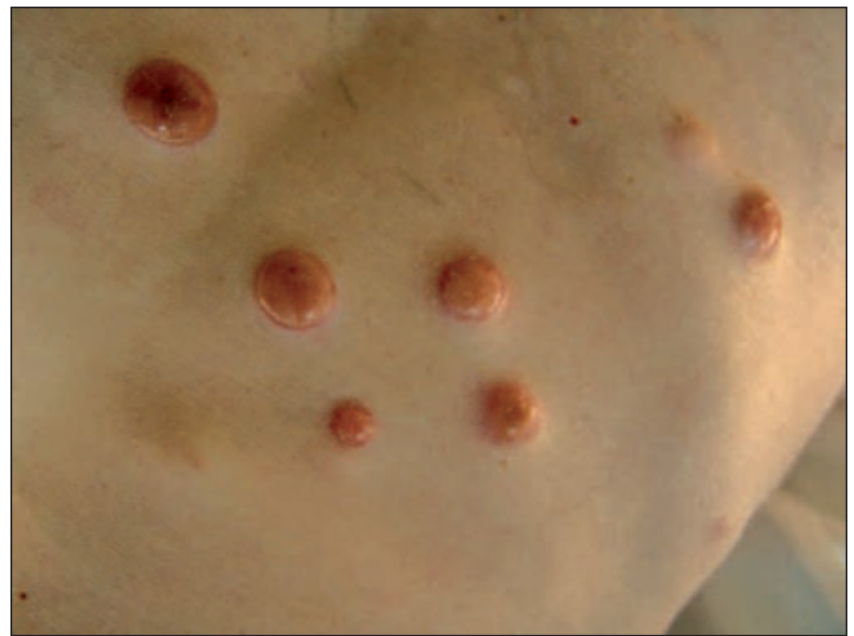

Fig. 3. Image of the patient's torso showing pigmented skin nodules. 
Malignant melanoma is the commonest tumour to metastasize to the gastrointestinal tract (GIT). Most frequent GIT metastasis includes the small bowel (50\%), colon $(31.3 \%)$ and anorectum $(25 \%)$ (1). Despite the frequent autopsy findings of GIT involvement by melanoma, the antemortem diagnosis is made only in 1.5 to $4.4 \%$ of all patients with melanoma (2).

The vast majority of GIT melanoma is metastatic from a cutaneous primary melanoma, although primary neoplasia can also arise from GIT (3).

Despite innocuous gastrointestinal symptoms, metastatic melanoma should be a diagnostic consideration in any patient with melanoma.

Prognosis of metastatic melanoma is extremely poor. Studies suggest a mean survival time of patients with sys- temic metastases from melanoma approximately 6 to 8 months $(4,5)$.

\section{REFERENCES}

1. Blecker D, Abraham S, Furth EE, Kochman ML. Melanoma in the gastrointestinal tract. Am J Gastroenterol 1999; 94(12): 3427-33.

2. Kadivar TF, Vanek VW, Krishnan EU. Primary malignant melanoma of the small bowel: a case study. Am Surg 1992; 58(7): 418-22.

3. Schuchter L, Green R, Fraker D. Primary and metastatic diseases in malignant melanoma of the gastrointestinal tract. Curr Opin Oncol 2000; 12: 181-5.

4. Barth A, Wanek LA, Morton DL. Prognostic factors in 1,521 melanoma patients with distant metastases. J Am Coll Surg 1995; 181: 193201.

5. Ollila DW, Essner R, Wanek LA, Morton DL. Surgical resection for melanoma metastatic to the gastrointestinal tract. Arch Surg 1996; 131: $975-80$. 